\title{
Characteristic Structural Knowledge for Morphological Identification and Classification in Meso-Scale Simulations Using Principal Component Analysis
}

\author{
Natthiti Chiangraeng ${ }^{1}$ (D), Michael Armstrong ${ }^{1}$, Kiattikhun Manokruang ${ }^{1}$ (D), Vannajan Sanghiran Lee ${ }^{2,3}$, \\ Supat Jiranusornkul ${ }^{4}$ and Piyarat Nimmanpipug ${ }^{1,3, *}$ D \\ 1 Department of Chemistry, Faculty of Science, Chiang Mai University, Chiang Mai 50200, Thailand; \\ natthiti.c@gmail.com (N.C.); armstrongmichael119@gmail.com (M.A.); kiattikhun.m@cmu.ac.th (K.M.) \\ 2 Department of Chemistry, Faculty of Science, University of Malaya, Kuala Lumpur 50603, Malaysia; \\ vannajan@um.edu.my \\ 3 Center of Excellence for Innovation in Analytical Science and Technology (I-ANALY-S-T), \\ Chiang Mai University, Chiang Mai 50200, Thailand \\ 4 Department of Pharmaceutical Sciences, Chiang Mai University, Chiang Mai 50200, Thailand; \\ supat.jira@cmu.ac.th \\ * Correspondence: piyarat.n@cmu.ac.th
}

\section{check for}

updates

Citation: Chiangraeng, N.; Armstrong, M.; Manokruang, K.; Lee, V.S.; Jiranusornkul, S.; Nimmanpipug, P. Characteristic Structural Knowledge for Morphological Identification and Classification in Meso-Scale Simulations Using Principal Component Analysis. Polymers 2021, 13, 2581. https:// doi.org/10.3390/polym13162581

Academic Editor: Riccardo Concu

Received: 30 June 2021

Accepted: 2 August 2021

Published: 4 August 2021

Publisher's Note: MDPI stays neutral with regard to jurisdictional claims in published maps and institutional affiliations.

Copyright: (c) 2021 by the authors. Licensee MDPI, Basel, Switzerland. This article is an open access article distributed under the terms and conditions of the Creative Commons Attribution (CC BY) license (https:/ / creativecommons.org/licenses/by/ $4.0 /)$.
Abstract: Meso-scale simulations have been widely used to probe aggregation caused by structural formation in macromolecular systems. However, the limitations of the long-length scale, resulting from its simulation box, cause difficulties in terms of morphological identification and insufficient classification. In this study, structural knowledge derived from meso-scale simulations based on parameters from atomistic simulations were analyzed in dissipative particle dynamic (DPD) simulations of PS- $b$-PI diblock copolymers. The radial distribution function and its Fourier-space counterpart or structure factor were proposed using principal component analysis (PCA) as key characteristics for morphological identification and classification. Disorder, discrete clusters, hexagonally packed cylinders, connected clusters, defected lamellae, lamellae and connected cylinders were effectively grouped.

Keywords: polystyrene; polyisoprene; morphology; copolymer; structure factor; PCA

\section{Introduction}

Self-assembly of block copolymers resulting in an order-structural formation in polymeric materials has been extensively studied due to the wide range of potential applications [1,2]. Diblock copolymers comprised of chemically incompatible blocks that consist of only two dissimilar blocks bound together have attracted much attention due to the fact that, despite their simple chemical structures, they can give rise to rich morphological behavior [3-5]. Sphere-liked micelles, cylinders, gyroids and lamellae are the characteristically observed morphologies [3-6].

A target morphology and its potential applications were investigated from an experimental point of view and confirmed using various characterization techniques [7-9], including vibrational spectroscopy (IR/Raman), differential scanning calorimetry (DSC), scanning electron microscopy (SEM), transmission electron microscopy (TEM), wide-angle X-ray diffraction (WAXD), small-angle X-ray scattering (SAXS), small-angle neutron scattering (SANS), etc. Complementary results were also utilized to achieve the goal. Weiyu et al. reported that typical morphologies of polyethylene-block-polyethylene oxide (PE- $b$ PEO) were successfully identified using the previously mentioned scientific instruments and that the temperature was responsible for their morphological transitions [9]. Morphological changes from lamellae structures to gyroid, hexagonally packed, cylindrical and spherical structures due to temperature were revealed and discussed in detail for the 
case of $\mathrm{PE}_{17}-b-\mathrm{PEO}_{40}$. The authors also reported that the changes in these components during morphological transformations could be varied because of their different intrinsic properties, such as temperature responsiveness and so forth. Khandpur and co-workers used a combination of dynamic mechanical spectroscopy (DMS), TEM, SEM and SANS to indicate and classify morphologies of polystyrene-block-polyisoprene (PS-b-PI) copolymers with PI volume fractions ranging from 0.24 to 0.82 [3,4]. The study showed that ordinary morphologies can be detected and observed. The phase diagram was constructed to show a possible region in which a particular morphology could be observed. The experimental result was in line with the theoretical study undertaken by Cochran and co-workers [10].

Modeling tools can be used to successfully visualize a morphology obtained from simulations. Nevertheless, it can be problematic to distinguish irregular morphologies with intricate structures in the complex arrangements. Typically, an order parameter is utilized to distinguish the appearance of the simulated morphologies. This function is often implemented in computational routines in order to be useful in material modeling. In dynamic mean-field density functional (DDF) [11,12] theory, a transformation of morphology from the beginning to the end of simulation can be investigated using order parameters defined in terms of the mean squared deviation from homogeneity in volume $V[6,13]$. The order parameter is given by

$$
\text { order parameter }=\frac{1}{V} \int_{V}\left[\eta_{i}^{2}(r)-\eta_{i}^{2}\right] d r,
$$

where $\eta_{i}$ is the dimensionless density for species $i$. The tendency of the order parameters can be used to indicate the transformation of morphologies from one to another as well as to confirm the stable morphology. A high value of the order parameter refers to possible phase segregation, whereas components are more miscible when the value is close to zero [13]. Another coarse-grained simulation is the dissipative particle dynamic (DPD) approach implemented in the DL_MESO software package [14]. An output gives three different eigenvalues over a simulation time. The isosurface normal distribution $p(\mathbf{n})$ can be constructed using the obtained densities and the average value of the overall density, which is used as the isosurfaces' threshold. The second moment or symmetric tensor $\mathbf{M}$ is written as

$$
\mathbf{M}=\int \mathbf{n n} p(\mathbf{n}) \mathrm{d} \mathbf{n},
$$

in which $\mathbf{M}$ describes an ideal of how the particles are distributed and arranged in the system. The solution of this equation provides three eigenvalues $\mu_{i}$, where $\sum_{i=1}^{3} \mu_{i}=1$ can be used as the order parameter for morphological identification and classification similarly as described above $[15,16]$. This order parameter can distinguish a type of morphology into three main mesophases, including isotropic $\left(\mu_{1} \approx \mu_{2} \approx \mu_{3}\right)$, cylindrical $\left(\mu_{1} \ll \mu_{2}, \mu_{3}\right)$ and lamellar $\left(\mu_{1}, \mu_{2} \ll \mu_{3}\right)$ mesophases [5]. The criteria used to judge here were proposed by Preinsen et al. [15] and Warren et al. [16] However, the arrangements of some morphologies are too sophisticated to distinguish solely using a visualizer or order parameter, as described in our previous paper [5].

To overcome this limitation, principal component analysis (PCA) is introduced and utilized in this study. PCA is a suitable method because it can reduce dimensionality in the datasets into a few principal components that are explainable and understandable [17]. Each data point can be plotted and can display the cluster of the calculated datasets. The contribution of each classification along with the squared cosine $\left(\cos ^{2}\right)$, or the quality of representations, can provide a visual explanation of how important the data are in each classification and how much data are compressed in each component [18]. It can generate a set of new variables, namely principal components (PCs), to represent datasets of interest. This algorithm aims to reduce the dimensionality of a dataset to emphasize the main and 
important variations in the data. With the PCA algorithm, an original matrix $(X)$ of the dataset can be transformed into two new matrices, which is given by

$$
X=T P+E,
$$

where $T$ is a score matrix, $P$ is a loading matrix and $E$ is a residual containing the variation. The score plot provides the location of the samples and the loading plot indicates correlations among variables. PCA has successfully been used to project a mean squared displacement (MSD) in datasets analyzed from simulation trajectories to explain the mechanistic insights of biological molecules [19]. Fernandez and coworkers revealed that the projection of the datasets of the atomic property-weighted radial distribution functions (RDFs) to the first and second principal components can successfully distinguish geometrical properties and gas uptake capacities of metal-organic frameworks [20].

In light of this information, we realized that an improvement to the method for classification of a simulated morphology type was necessary and challenging. The developed method could be a valuable and useful tool for other researchers to identify and classify an observed morphology easily, precisely, and accurately by using PCA. In this study, morphologies of PS- $b$-PI diblock copolymer were explored using DPD simulations. A suitable condition that gives a variety of morphologies was selected from our previous paper [5] as a demonstration. PCA was applied to distinguish the significance of morphologies that are difficult to identify using only physical appearance and order parameters. The sets of structural properties were used as crucial variables in the PCA. The observed quantitative data are useful for the classification of morphologies correctly.

\section{Methods}

2.1. Dissipative Particle Dynamics (DPD) Simulation and the Analysis of an Apparent Morphology at an Equilibrium and Its Related Order Parameters

Chain assembly of polystyrene-block-polyisoprene (PS-b-PI) diblock copolymer was explored by means of dissipative particle dynamics simulations. The elementary unit in DPD is a spherical particle representing a fluid element, which in turn represents linearly connected chains composed of S- and I-type DPD particles for styrene and isoprene, respectively. The molecular structures and their corresponding coarse-grained models for both types of DPD particles are illustrated in Figure 1. All boxes contained 24,000 coarsegrained particles with a density $\sigma$ of 3.0. Each particle was connected by a spring with a constant $C_{i j}$ of 4 . The additional parameters are provided in Table S1. The primitive coarsegrained structures reported in reference [5] were used and created using the moleculegeneration tool (molecule.exe) in the DL_MESO software (2.7 rev 08, Daresbury Laboratory, Daresbury, UK) package [14]. We selected the coarse-grained models $\mathrm{S}_{1} \mathrm{I}_{19}, \mathrm{~S}_{2} \mathrm{I}_{18}, \mathrm{~S}_{3} \mathrm{I}_{17}$, $\mathrm{S}_{4} \mathrm{I}_{16}, \mathrm{~S}_{5} \mathrm{I}_{15}, \mathrm{~S}_{6} \mathrm{I}_{14}, \mathrm{~S}_{7} \mathrm{I}_{13}, \mathrm{~S}_{8} \mathrm{I}_{12}, \mathrm{~S}_{9} \mathrm{I}_{11}, \mathrm{~S}_{10} \mathrm{I}_{10}, \mathrm{~S}_{11} \mathrm{I}_{9}, \mathrm{~S}_{12} \mathrm{I}_{8}, \mathrm{~S}_{13} \mathrm{I}_{7}, \mathrm{~S}_{14} \mathrm{I}_{6}, \mathrm{~S}_{15} \mathrm{I}_{5}, \mathrm{~S}_{16} \mathrm{I}_{4}, \mathrm{~S}_{17} \mathrm{I}_{3}, \mathrm{~S}_{18} \mathrm{I}_{2}$ and $S_{19} I_{1}$ with a volume fraction range from 0.05 to 0.95 . This condition was chosen because of the variety of simulated morphologies observed in a symmetric phase diagram [5] carried out at $393 \mathrm{~K}$, which corresponded well with the experimental observations by Khandpur and co-workers [3,4]. In this DPD simulation, each system was firstly equilibrated for $1,000,000$ steps with a step size of 0.01 . Subsequently, a production run was carried out until a morphology reached an equilibrium state. All DPD simulations were carried out using the DL_MESO software package. 


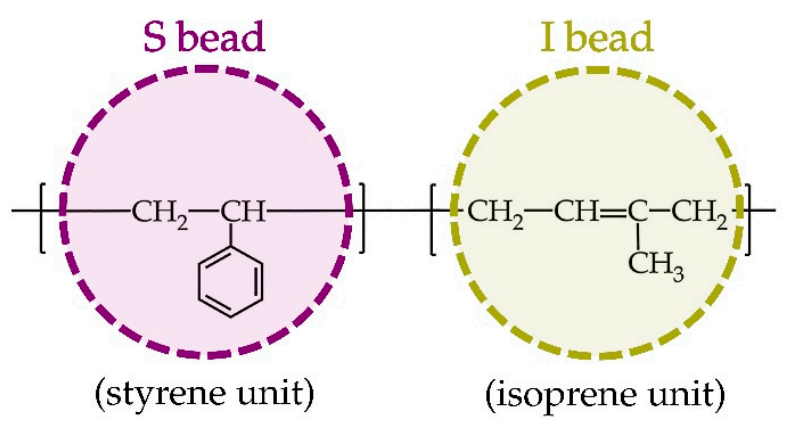

Figure 1. Chemical structures of styrene and isoprene units and their corresponding coarse-grained beads in the simulation system.

For data manipulations, we used export_image_vtf.exe to extract the final structure of coarse-grained structures and subsequently used VMD software (1.9.3, Theoretical and Computational Biophysics Group, Urbana, IL, USA) [21] to convert a structure into a pdb format file for visualization. ParaView [22] was used to visualize the structure of the coarse-grained beads, the isodensity of each component with a color gradient and an isosurface showing a surface between the different species in a simulation. The program isosurfaces.exe was utilized to extract a file in the VTK format for visualization of the isodensity and isosurface, which were calculated to the order parameter. The radial distribution function (RDF) and its Fourier-space counterpart, $S(k)$, were computed using the embedded rdfmol.exe tool in DL_MESO software. In this study, the last 1,000,000 simulation steps from each model were used for the analysis. The proposed methodology employed entirely free-to-use software available to academic and non-commercial sectors.

\subsection{Principal Component Analysis (PCA)}

In this study, PCA analysis was performed and visualized using R programming language [23]. All calculations were performed using available built-in packages. The RDF and $S(k)$ datasets of 19 different coarse-grained models providing 7 distinct morphologies were coded as variables. In this work, a range of radius in DPD units from 0.085 to 9.995 was selected for the set of RDF data while a range of $k$ from 0.30 to 315.00 was chosen for the set of $S(k)$ data. In the calculation of the PCA, a selected range of RDF and $S(k)$ values were separately designated as individual data points for each variable. Before performing the PCA, we checked the distribution of the data points to ensure that there was a major contribution into the first two principal components (PC1 and PC2). Then, we carried out full PCA calculations.

The Factoextra [24] and Corrplot [25] packages were additionally used for visualizing the PCA results. The Factoextra package was used to generate the loading plots and to show a classification of variables of interest. The Corrplot package was used to generate a correlation plot of their contributions and quality values in order to observe the compression of each variable. The size of the circle and the color shade in this plot represent the contribution and quality in each PC, respectively. The eigenvalues of the quality were used to indicate the quality of representations. Using this package, such values are typically normalized to a value of -1 to 1 and represented by a color gradient. The PCA script for structural knowledge analysis and data visualization was provided in Script S1.

\section{Results}

3.1. Radial Distribution Function (RDF) and Structure Factor ( $S(k))$ Analysis of Coarse-Grained Structures

In order to investigate the equilibrium morphologies of the PS- $b$-PI copolymer, the copolymeric chains were mapped onto coarse-grained beads by comparing the specific volume of isoprene or styrene to that of water molecules. In our previous study we reported that the isoprene or styrene monomer is approximately equivalent to a single coarse-grained 
bead [5]. This finding is also consistent with the results reported by Li and coworkers [26]. In this study, 19 coarse-grained models were constructed based on our previous study [5], specifically $\mathrm{S}_{1} \mathrm{I}_{19}, \mathrm{~S}_{2} \mathrm{I}_{18}, \mathrm{~S}_{3} \mathrm{I}_{17}, \mathrm{~S}_{4} \mathrm{I}_{16}, \mathrm{~S}_{5} \mathrm{I}_{15}, \mathrm{~S}_{6} \mathrm{I}_{14}, \mathrm{~S}_{7} \mathrm{I}_{13}, \mathrm{~S}_{8} \mathrm{I}_{12}, \mathrm{~S}_{9} \mathrm{I}_{11}, \mathrm{~S}_{10} \mathrm{I}_{10}, \mathrm{~S}_{11} \mathrm{I}_{9}, \mathrm{~S}_{12} \mathrm{I}_{8}, \mathrm{~S}_{13} \mathrm{I}_{7}$, $\mathrm{S}_{14} \mathrm{I}_{6}, \mathrm{~S}_{15} \mathrm{I}_{5}, \mathrm{~S}_{16} \mathrm{I}_{4}, \mathrm{~S}_{17} \mathrm{I}_{3}, \mathrm{~S}_{18} \mathrm{I}_{2}$ and $\mathrm{S}_{19} \mathrm{I}_{1}$. After that, their equilibrium morphologies were explored through DPD simulations. From the DPD simulations, the coarse-grained models aggregated into a variety of characteristic morphologies at a simulation temperature of $393 \mathrm{~K}$.

Using an interchain RDF analysis, the morphologies both with similar or different physical arrangements were obviously noticed, as shown in Figure 2. The overlapping graphs indicated that the arrangements of coarse-grained beads were very similar and, vice versa, that the morphologies were different. The criteria to distinguish the similarity of the morphologies were a magnitude of $\mathrm{g}(r)$ and a graph shape.

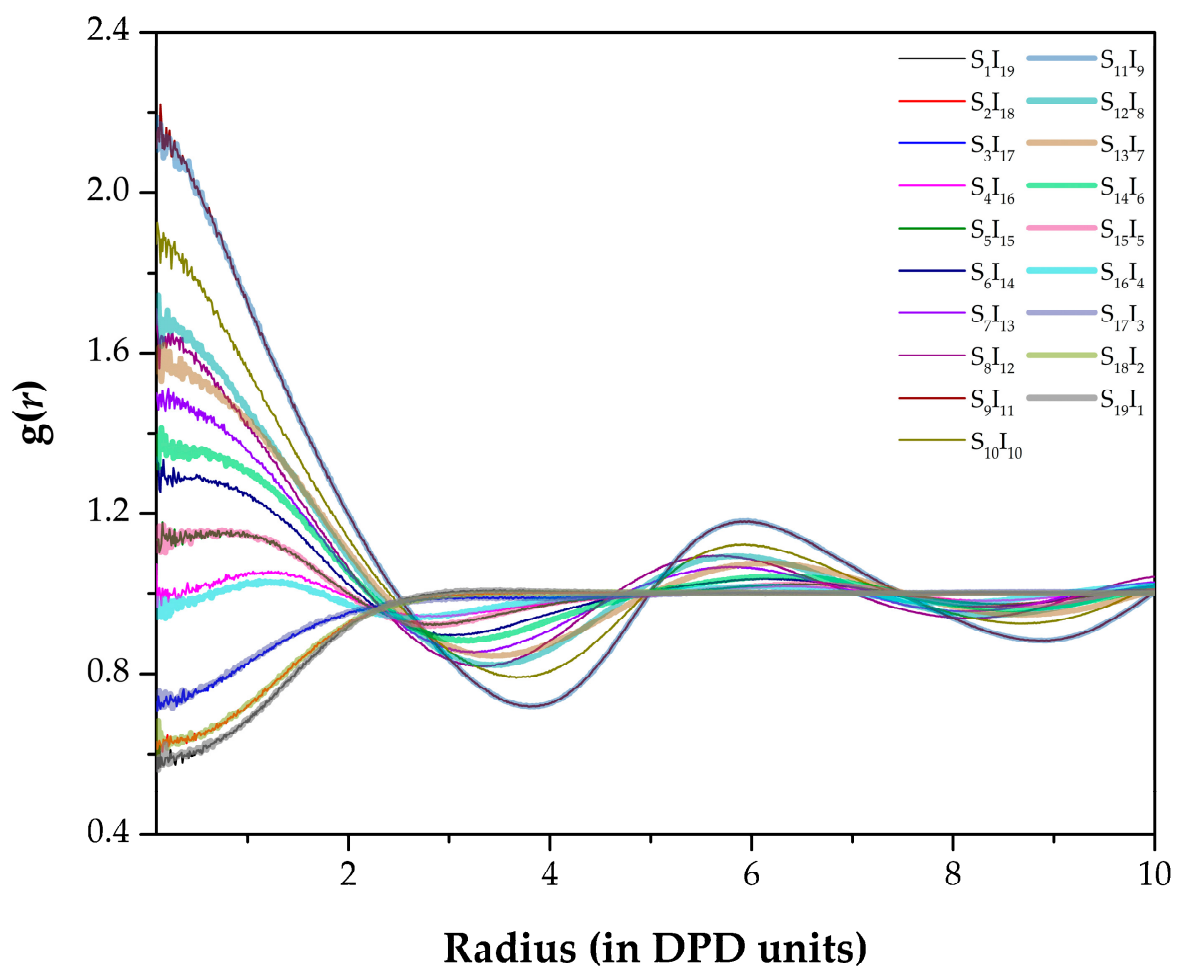

Figure 2. RDFs $g(r)$ between chain-chain types in different coarse-grained models. $r$ is plotted in the reduced DPD length unit. Some lines are bolded and transparent to ensure that all data can be seen.

The structure factor is an experimentally observable parameter detected via a scientific instrument, such as the X-ray diffraction technique. This parameter depends on an arrangement of molecular structures in a material of interest and it is a useful method to distinguish the similarity or dissimilarity of materials, as has been reported in various papers $[7,9,27,28]$. $\mathrm{S}(k)$ can be understood in a similar way as the explanation of the RDF graph above. It was successfully used to classify the types of simulated morphologies, as illustrated in Figure 3. However, by solely using RDFs and $S(k)$, one cannot group each morphology into the three main categories (isotropic, hexagonal and lamellar mesophases), as suggested by the order parameter. Therefore, a PCA analysis was carried out in this study and is discussed in the last section. 


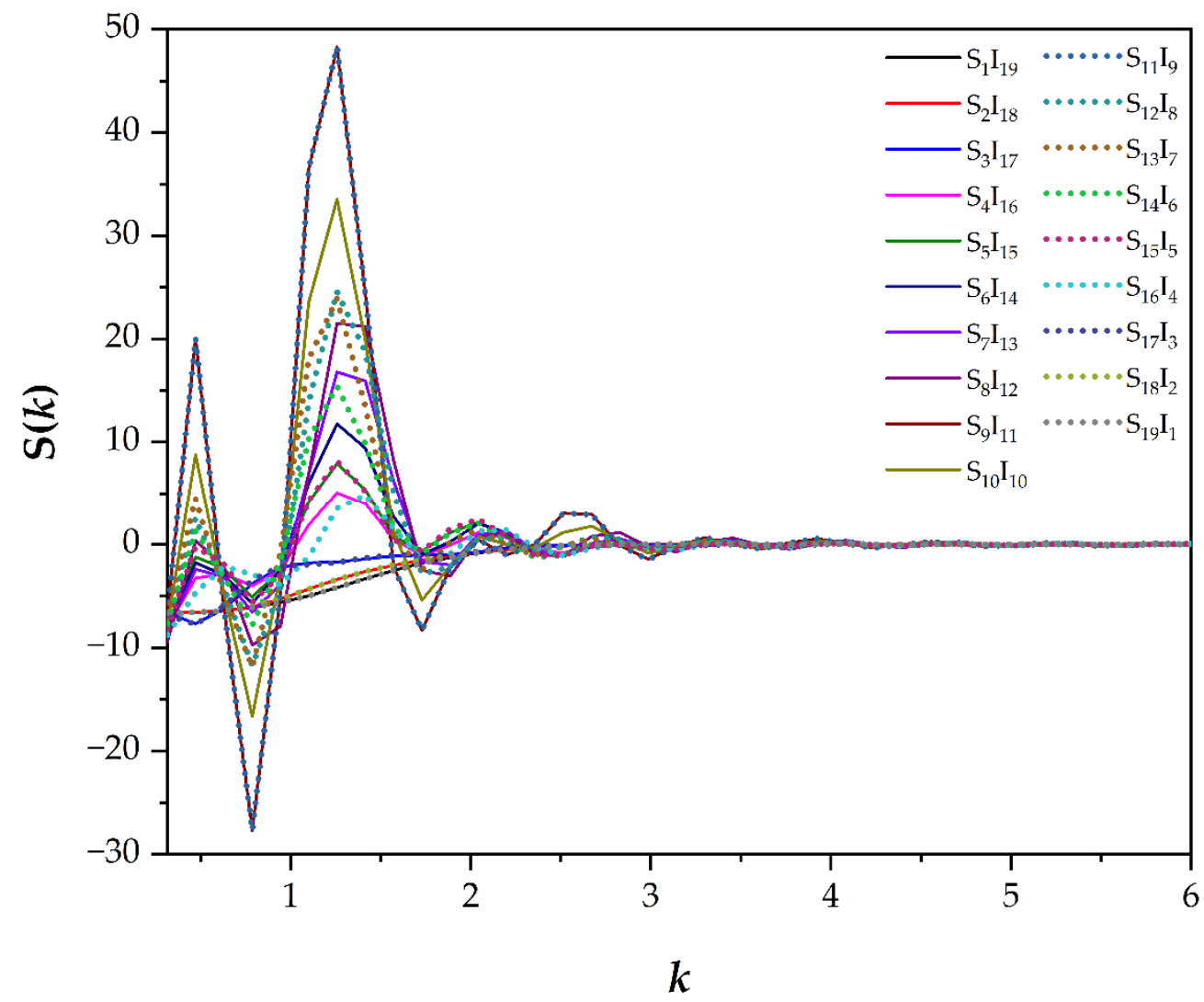

Figure 3. Structure factors $S(k)$ in different coarse-grained models versus a frequency $k$. Some lines are illustrated in a dotted style to ensure that all data can be seen.

In this study, the $\mathrm{S}(k)$ of a simulated gyroidal morphology corresponded well to the experimentally reported data of the results detected by the X-ray diffraction technique. The peak position ratio of the experimentally observable refractions contained $\sqrt{3}, \sqrt{4}$, $\sqrt{7}, \sqrt{8}, \sqrt{10}, \sqrt{11}, \sqrt{12}, \sqrt{13}, \sqrt{15}$ and $\sqrt{16}$ [27]. The peaks appeared in our study as shown in Figure 4 and consisted of $\sqrt{2}, \sqrt{4}, \sqrt{7}, \sqrt{8}, \sqrt{10}, \sqrt{12}, \sqrt{15}$ and $\sqrt{16}$. The first peak slightly shifted to $\sqrt{2}$ and the peaks at $\sqrt{11}$ and $\sqrt{13}$ were missing from the simulated $\mathrm{S}(k)$; these observations could be a distortion of the gyroid. For example, two gyroidal morphologies obtained from $\mathrm{S}_{6} \mathrm{I}_{14}$ and $\mathrm{S}_{14} \mathrm{I}_{6}$ gave slight differences in the simulated $\mathrm{S}(k)$ due to the different structures, as shown in Figure 4 and Figure S1, respectively.

\subsection{Principal Component Analysis (PCA) for Classification of Morphologies}

Using the built-in packages, all classifications in the RDF and $S(k)$ datasets were compressed into the first two components with a cumulative variance of $98.17 \%$ and $94.59 \%$, respectively, as shown in Table 1 . The correlation plots of each dataset (Figure 5) show that most of the data was compressed into the first two components, which matches with the corresponding cumulative variance percentages. In the RDF dataset (Figure 5a), $\mathrm{S}_{4} \mathrm{I}_{16}$ contributed to the two components equally. $\mathrm{S}_{16} \mathrm{I}_{4}$ mostly contributed to PC2 while the rest of the classifications were mostly compressed into PC1. In the $S(k)$ dataset (Figure $5 b$ ), the datasets of $\mathrm{S}_{4} \mathrm{I}_{16}$ to $\mathrm{S}_{16} \mathrm{I}_{4}$ were mostly compressed into PC1, while the rest contributed to PC2. 


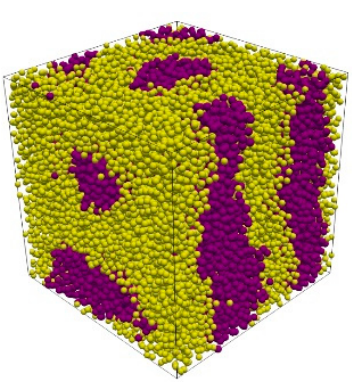

(a)

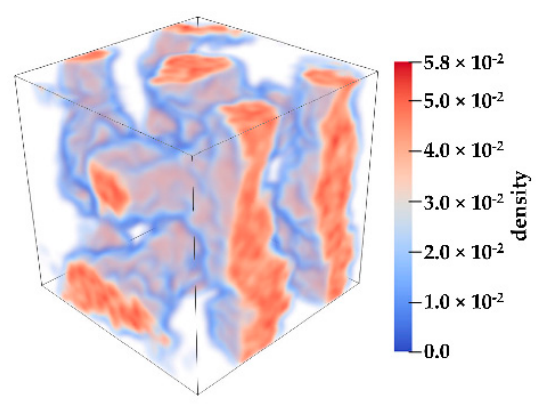

(c)

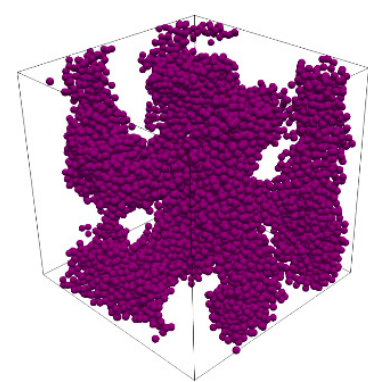

(b)

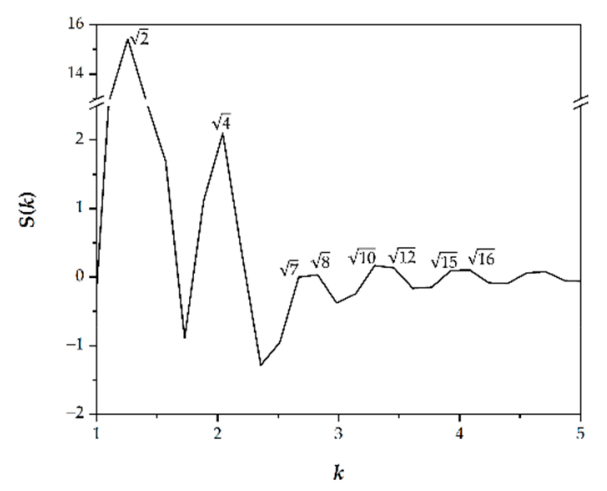

(d)

Figure 4. The coarse-grained model obtained from an $\mathrm{S}_{14} \mathrm{I}_{6}$ : (a) an arrangement of the styrene (yellow) and isoprene (violet); (b) isoprene beads resulting solely in a skeleton of gyroidal morphology; and (c) a gradient of styrene density. The red regions correspond to high densities of isoprene and vice versa for the blue region. (d) The simulated $S(k)$.

Table 1. Eigenvalue and accumulated contribution ratio for the principal components (PCs) using RDF and S(k) data.

\begin{tabular}{ccccccc}
\hline & \multicolumn{3}{c}{ RDFs } & & \multicolumn{2}{c}{$\mathbf{S}(\boldsymbol{k})$} \\
\cline { 2 - 7 } PC No. & Eigenvalue & $\begin{array}{c}\text { Variance } \\
\text { Percent(\%) }\end{array}$ & $\begin{array}{c}\text { Accumulated } \\
\text { Variance Percent (\%) }\end{array}$ & Eigenvalue & $\begin{array}{c}\text { Variance } \\
\text { Percent (\%) }\end{array}$ & $\begin{array}{c}\text { Accumulated } \\
\text { Variance Percent (\%) }\end{array}$ \\
\hline 1 & $1.67 \times 10^{1}$ & 87.94 & 87.94 & $1.13 \times 10^{1}$ & 59.69 & 59.69 \\
2 & 1.94 & 10.23 & 98.17 & 6.63 & 34.89 & 94.59 \\
3 & $2.15 \times 10^{-1}$ & 1.13 & 99.30 & $6.27 \times 10^{-1}$ & 3.30 & 97.88 \\
4 & $6.93 \times 10^{-2}$ & 0.36 & 99.67 & $2.14 \times 10^{-1}$ & 1.13 & 99.01 \\
5 & $3.05 \times 10^{-2}$ & 0.16 & 99.83 & $1.61 \times 10^{-1}$ & 0.85 & 99.86 \\
6 & $1.52 \times 10^{-2}$ & 0.08 & 99.91 & $2.36 \times 10^{-2}$ & 0.12 & 99.99 \\
7 & $6.31 \times 10^{-3}$ & 0.03 & 99.94 & $1.90 \times 10^{-3}$ & 0.01 & 100.00 \\
8 & $2.71 \times 10^{-3}$ & 0.01 & 99.95 & $5.77 \times 10^{-4}$ & 0.00 & 100.00 \\
9 & $2.33 \times 10^{-3}$ & 0.01 & 99.97 & $2.66 \times 10^{-4}$ & 0.00 & 100.00 \\
10 & $2.15 \times 10^{-3}$ & 0.01 & 99.98 & $2.55 \times 10^{-5}$ & 0.00 & 100.00 \\
11 & $1.13 \times 10^{-3}$ & 0.01 & 99.98 & $6.06 \times 10^{-6}$ & 0.00 & 100.00 \\
12 & $8.03 \times 10^{-4}$ & 0.00 & 99.99 & $4.77 \times 10^{-6}$ & 0.00 & 100.00 \\
13 & $6.66 \times 10^{-4}$ & 0.00 & 99.99 & $1.28 \times 10^{-6}$ & 0.00 & 100.00 \\
14 & $4.80 \times 10^{-4}$ & 0.00 & 99.99 & $8.82 \times 10^{-7}$ & 0.00 & 100.00 \\
15 & $3.11 \times 10^{-4}$ & 0.00 & 100.00 & $5.52 \times 10^{-7}$ & 0.00 & 100.00 \\
16 & $2.92 \times 10^{-4}$ & 0.00 & 100.00 & $4.21 \times 10^{-7}$ & 0.00 & 100.00 \\
17 & $2.39 \times 10^{-4}$ & 0.00 & 100.00 & $2.44 \times 10^{-7}$ & 0.00 & 100.00 \\
18 & $1.90 \times 10^{-4}$ & 0.00 & 100.00 & $2.05 \times 10^{-7}$ & 0.00 & 100.00 \\
19 & $1.26 \times 10^{-4}$ & 0.00 & 100.00 & $2.64 \times 10^{-8}$ & 0.00 & 100.00 \\
\hline
\end{tabular}




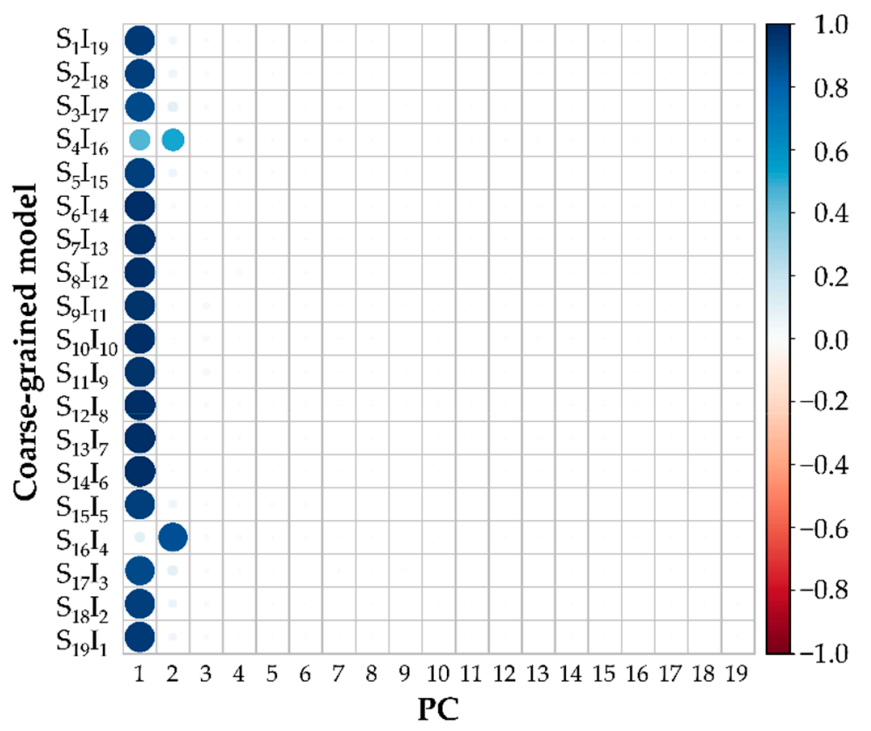

(a)

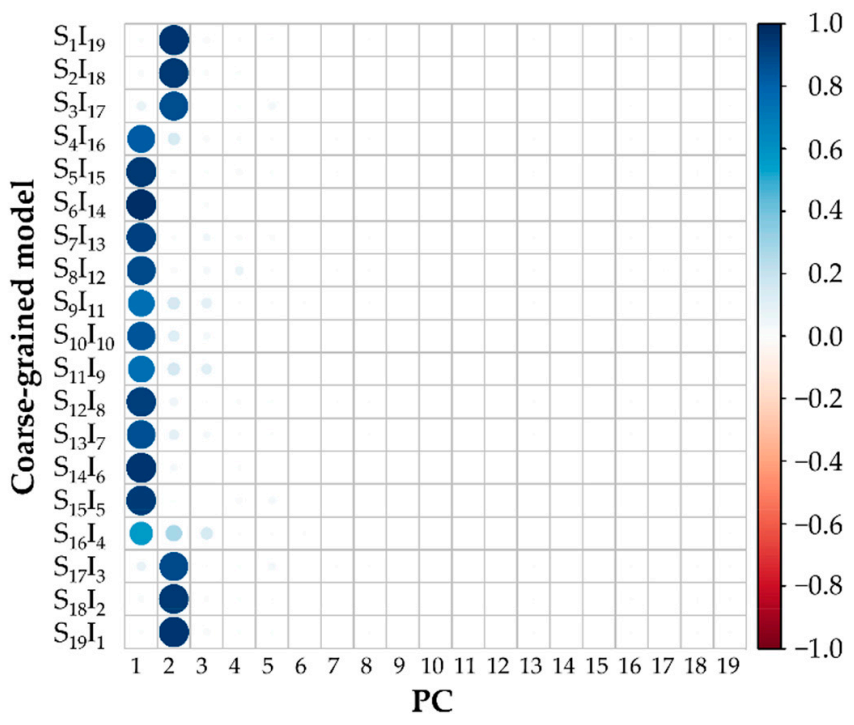

(b)

Figure 5. Correlation plots of (a) RDFs and (b) S(k) show a relationship between the variables of coarse-grained models and each principal component (PC). The diameter of each circle indicates the contribution of each variable in each PC. The color shades indicate the quality of each variable in each PC and the range of the quality is represented as a color gradient at the right-hand side.

According to the DPD simulations, aggregations were only affected by their different ratios with the monomeric units of each block. To classify the obtained morphologies, we investigated them using three routes: (1) the apparent aggregation of coarse-grained beads, which is illustrated on the left-hand side of each sub-figure in Figure 6; (2) an isosurface investigation, which is shown in the middle of the sub-figure in Figure 6; and (3) a consideration of order parameters calculated using an embedded tool in the DL_MESO package, which is illustrated on the right-hand side of each sub-figure in Figure 6. Route 1 is a typical way to visualize and classify an obtained morphology due to its simplicity, with no need for post-processing. The structure can also be directly visualized using the trajectory from a simulation. However, the drawbacks of the long-length scale, resulting from its simulation box, may cause difficulties in morphological identification.

For isosurface and order parameter assessments, further calculations had to be carried out using the program isosurfaces.exe. With this tool, the volume of particle was smeared using a Gaussian function with a standard deviation $\sigma$ :

$$
f(\mathrm{r})=\frac{1}{\left(2 \pi \sigma^{2}\right)^{\frac{3}{2}}} \exp \left(-\frac{\left|\mathrm{r}-\mathrm{r}_{\mathrm{i}}\right|^{2}}{2 \sigma^{2}}\right)^{\prime}
$$

where $\mathbf{r}_{i}$ is the position of particle $i$. All sampling points on a regular orthogonal grid within a distance of $3 \sigma$ can be obtained with this smearing function. The formations related to the densities of the system are written as a readable file for visualizing an isosurface in different species, which is considered as route 2 in the above visualization process.

According to Equation (4), the visualization of isosurfaces between different coarsegrained beads is very useful. We can see the real structure of each aggregation deep inside the structure, as shown in the middle of each sub-figure in Figure 6. The distinct morphologies are disorder, discrete clusters, hexagonally packed cylinders, connected clusters, defected lamellae, lamellae and connected cylinders, which were obtained from $\mathrm{S}_{1} \mathrm{I}_{19}, \mathrm{~S}_{2} \mathrm{I}_{18}, \mathrm{~S}_{3} \mathrm{I}_{17}, \mathrm{~S}_{4} \mathrm{I}_{16}, \mathrm{~S}_{5} \mathrm{I}_{15}, \mathrm{~S}_{6} \mathrm{I}_{14}, \mathrm{~S}_{7} \mathrm{I}_{13}, \mathrm{~S}_{8} \mathrm{I}_{12}, \mathrm{~S}_{9} \mathrm{I}_{11}$ and $\mathrm{S}_{10} \mathrm{I}_{10}$, respectively. The rich styrene models, $\mathrm{S}_{11} \mathrm{I}_{9}, \mathrm{~S}_{12} \mathrm{I}_{8}, \mathrm{~S}_{13} \mathrm{I}_{7}, \mathrm{~S}_{14} \mathrm{I}_{6}, \mathrm{~S}_{15} \mathrm{I}_{5}, \mathrm{~S}_{16} \mathrm{I}_{4}, \mathrm{~S}_{17} \mathrm{I}_{3}, \mathrm{~S}_{18} \mathrm{I}_{2}$ and $\mathrm{S}_{19} \mathrm{I}_{1}$, exhibited a 
similar morphology as the rich isoprene models, as shown in Figure S2. All the structures observed using a combination of the three routes for classification are listed in Table 2.
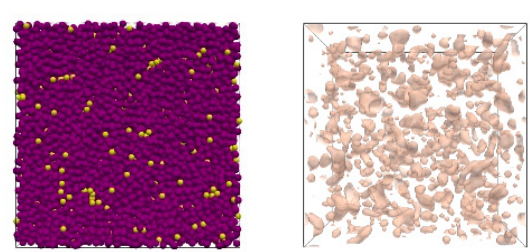

(a)
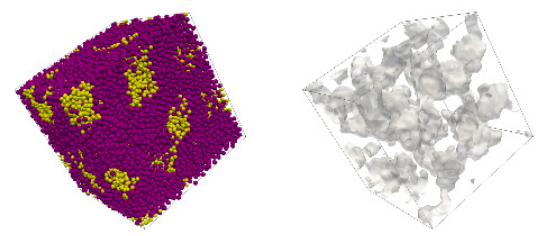

(c)
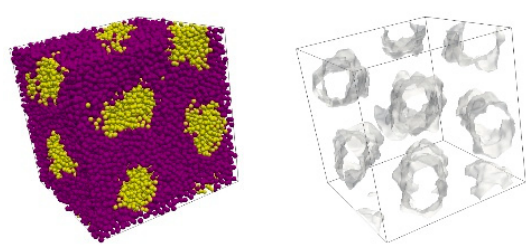

(e)
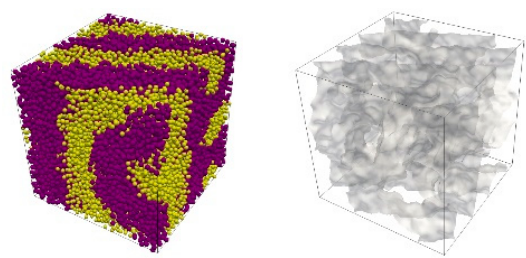

(g)
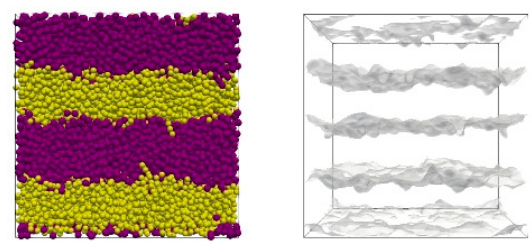

(i)
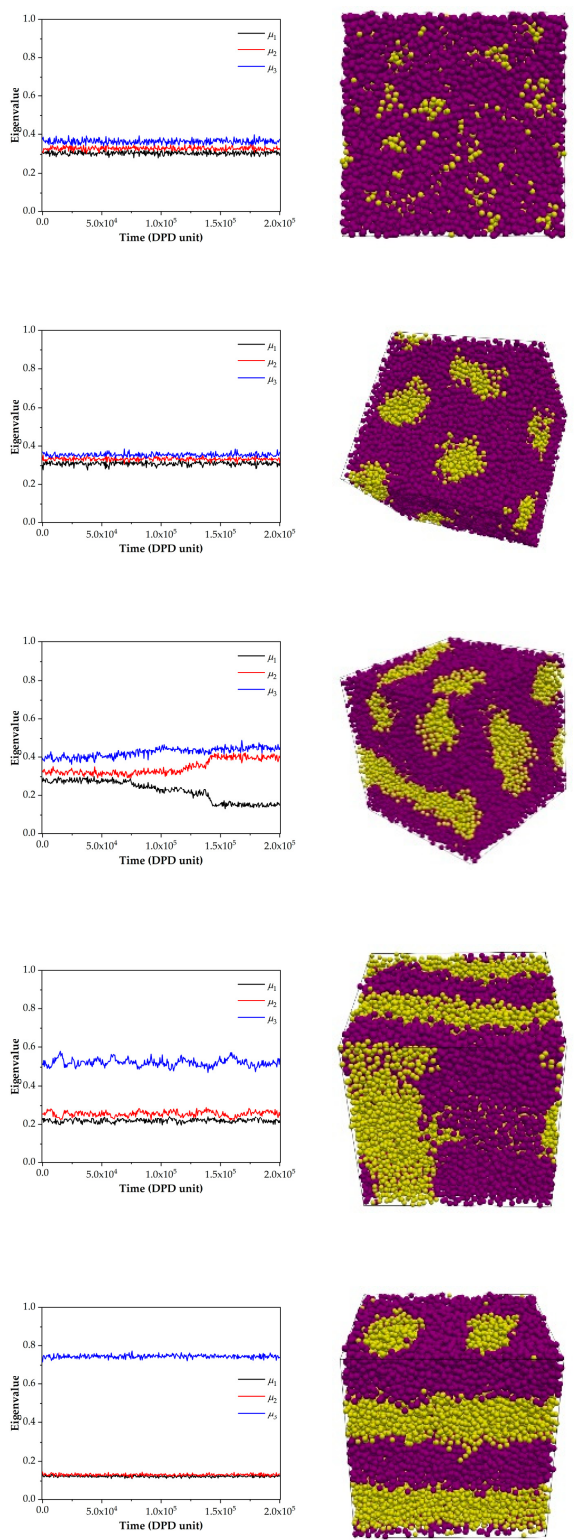
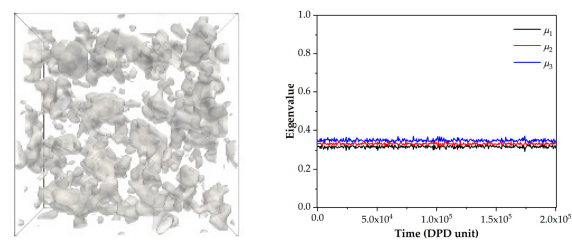

(b)

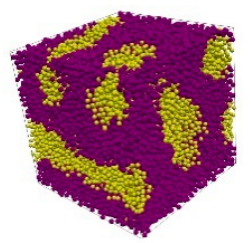

(d)

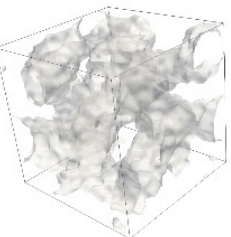

(f)
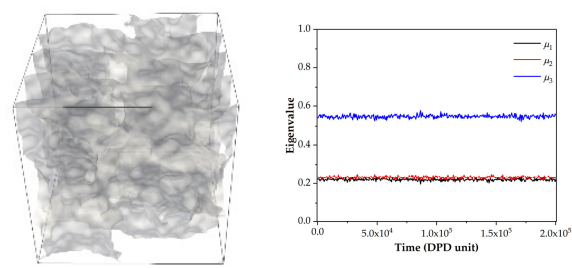

(h)
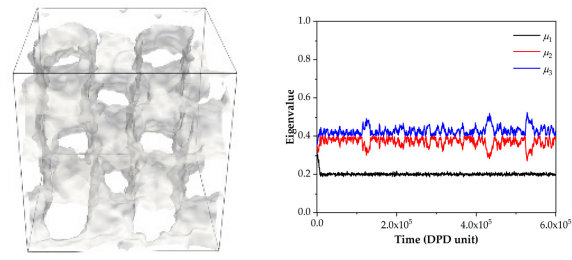

(j)

Figure 6. Panels in the composite figures illustrate apparent bead arrangements, isosurfaces between distinct bead types and the order-parameter sorting, from left to right, of (a) $\mathrm{S}_{1} \mathrm{I}_{19}$, (b) $\mathrm{S}_{2} \mathrm{I}_{18}$, (c) $\mathrm{S}_{3} \mathrm{I}_{17}$, , d ) $\mathrm{S}_{4} \mathrm{I}_{16}$, (e) $\mathrm{S}_{5} \mathrm{I}_{15}$, (f) $\mathrm{S}_{6} \mathrm{I}_{14}$, (g) $\mathrm{S}_{7} \mathrm{I}_{13}$, (h) $\mathrm{S}_{8} \mathrm{I}_{12}$, (i) $\mathrm{S}_{9} \mathrm{I}_{11}$ and (j) $\mathrm{S}_{10} \mathrm{I}_{10}$, respectively. The order parameters were calculated using an executable, namely isosuefaces.exe, an implemented tool in the DL_MESO software. Yellow and violet beads represent coarse-grained beads of styrene and isoprene, respectively.

The loading plots the morphologies (Figure 7) showed that each variable was grouped according to its morphology. Both lamellae (L1 and L2) classifications were almost identical to each other on both plots; hence, the eigenvector of the two morphologies was overlapping. The identical classification could also be observed for discrete clusters (DC1-DC2) and disorder (D1-D4, and D2-D3). The hexagonal packet cylinders (HPC1-4) cluster was also clustered in a distinct group in both plots. However, connected cylinders (CC) were presented differently in each plot. While CC was more similar to L1 and L2 in the 
RDF dataset (Figure 7a), the similarity of CC shifted to defected lamellae (DL) 4 in the $S(k)$ dataset (Figure $7 b$ ). Furthermore, DL1-4 could be distinctively grouped in the RDFs dataset, but DL was clustered with connected clusters (CC1 and CC2).

Table 2. Coarse-grained models and their morphological classification.

\begin{tabular}{ccc}
\hline Volume Fraction of Styrene & Coarse-Grained Model & Morphology (Its Abbreviation) \\
\hline 0.05 & $\mathrm{~S}_{1} \mathrm{I}_{19}$ & Disorder (D1) \\
0.10 & $\mathrm{~S}_{2} \mathrm{I}_{18}$ & Disorder (D2) \\
0.15 & $\mathrm{~S}_{3} \mathrm{I}_{17}$ & Discrete clusters (DC1) \\
0.20 & $\mathrm{~S}_{4} \mathrm{I}_{16}$ & Hexagonally packed cylinders (HPC1) \\
0.25 & $\mathrm{~S}_{5} \mathrm{I}_{15}$ & Hexagonally packed cylinders (HPC2) \\
0.30 & $\mathrm{~S}_{6} \mathrm{I}_{14}$ & Connected clusters (CC1) \\
0.35 & $\mathrm{~S}_{7} \mathrm{I}_{13}$ & Defected lamellae (DL1) \\
0.40 & $\mathrm{~S}_{8} \mathrm{I}_{12}$ & Defected lamellae (DL2) \\
0.45 & $\mathrm{~S}_{9} \mathrm{I}_{11}$ & Lamellae (L1) \\
0.50 & $\mathrm{~S}_{10} \mathrm{I}_{10}$ & Connected cylinders (CC) \\
0.55 & $\mathrm{~S}_{11} \mathrm{I}_{9}$ & Lamellae (L2) \\
0.60 & $\mathrm{~S}_{12} \mathrm{I}_{8}$ & Defected lamellae (DL3) \\
0.65 & $\mathrm{~S}_{13} \mathrm{I}_{7}$ & Defected lamellae (DL4) \\
0.70 & $\mathrm{~S}_{14} \mathrm{I}_{6}$ & Connected clusters (CC2) \\
0.75 & $\mathrm{~S}_{15} \mathrm{I}_{5}$ & Hexagonally packed cylinders (HPC3) \\
0.80 & $\mathrm{~S}_{16} \mathrm{I}_{4}$ & Hexagonally packed cylinders (HPC4) \\
0.85 & $\mathrm{~S}_{17} \mathrm{I}_{3}$ & Discrete clusters (DC2) \\
0.90 & $\mathrm{~S}_{18} \mathrm{I}_{2}$ & Disorder (D3) \\
0.95 & $\mathrm{~S}_{19} \mathrm{I}_{1}$ & Disorder (D4)
\end{tabular}

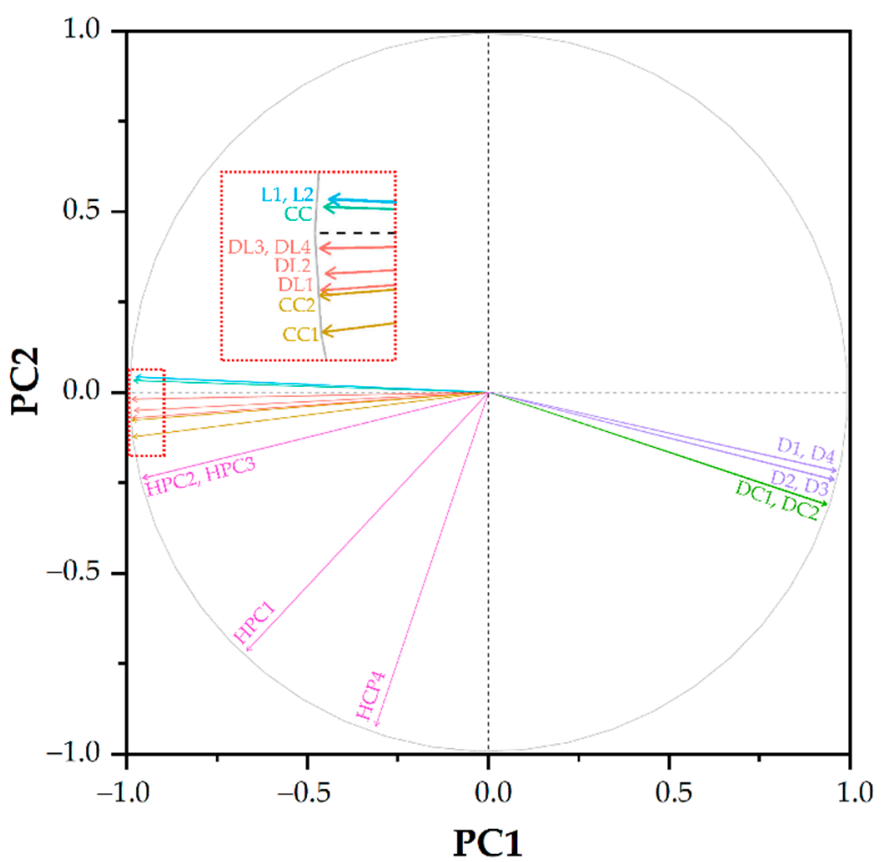

(a)

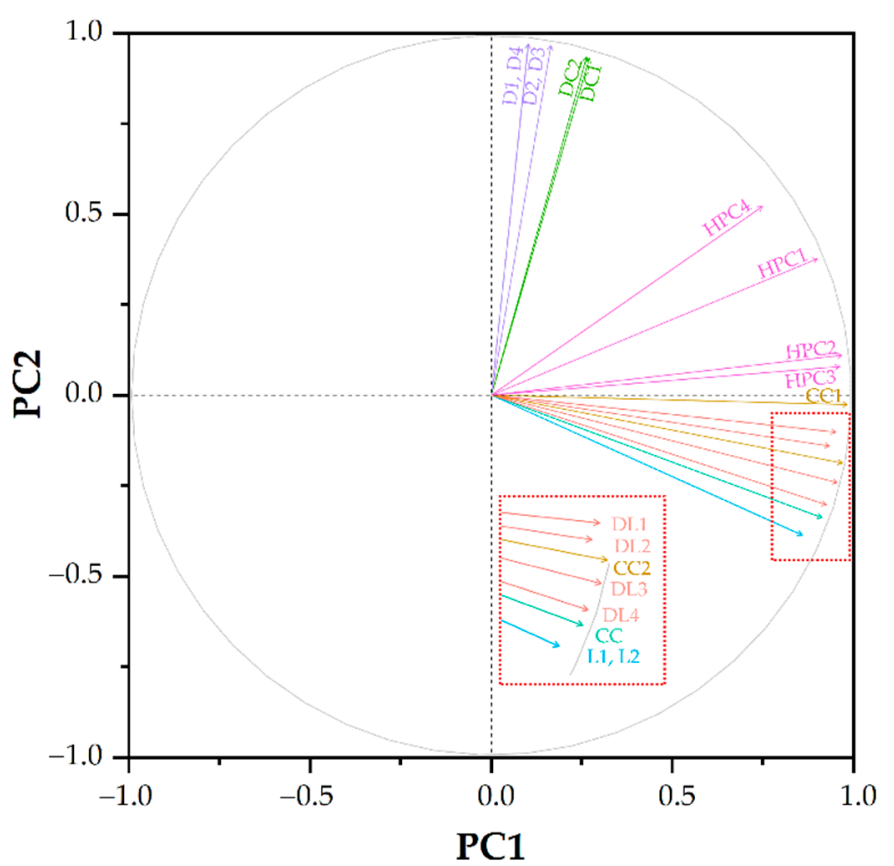

(b)

Figure 7. Loading plots of PCA analysis using (a) RDFs and (b) S(k) data. The colors violet, green, pink, yellow, red, blue and cyan correspond to disorder, discrete clusters, connected clusters, hexagonally packed cylinders, defected lamellae, lamellae and connected cylinders, respectively. The abbreviations are shown in Table 2.

\section{Conclusions}

Spatial intermolecular arrangement information derived from meso-scale simulations based on parameters from atomistic simulations were analyzed in dissipative particle dynamic (DPD) simulations of PS- $b$-PI diblock copolymers. In total, 19 coarse-grained models with different compositions were explored. The radial distribution function (RDF) 
and its Fourier-space counterpart, or structure factor $(\mathrm{S}(k))$, were proposed using PCA as key characteristics for morphological identification and classification. The RDF and $S(k)$ datasets were compressed into the first two components with a cumulative variance of $98.17 \%$ and $94.59 \%$, respectively. The eigenvalues of the second moment of the tensor built up from the normal vectors on the isosurface of the density function for each particle in the DPD simulation were used as a guideline in the phase determination. To complete this analysis when the classification of some morphologies was limited, i.e., connected clusters and connected cylinders, an add-on analysis using PCA was effectively applied in this system. By employing PCA to reduce the dimensionality of the RDF and its Fourier-space counterpart from DPD simulations, a complicated arrangement of morphologies found in PS- $b$-PI copolymers could be successfully differentiated. Disorder, discrete clusters, hexagonally packed cylinders, connected clusters, defected lamellae, lamellae and connected cylinders were effectively grouped.

Supplementary Materials: The following are available online at https:/ /www.mdpi.com/article/10 $.3390 /$ polym13162581/s1, Figure S1: Structure factors $\mathrm{S}(k)$ between chain-chain types of the gyroidal morphologies of $\mathrm{S}_{6} \mathrm{I}_{14}$ and $\mathrm{S}_{14} \mathrm{I}_{6}$; Figure S2: Panels in the composite figures illustrate apparent bead arrangements, isosurfaces between distinct bead types and order-parameter sorting, from left to right, of (a) $\mathrm{S}_{11} \mathrm{I}_{9}$, (b) $\mathrm{S}_{12} \mathrm{I}_{8}$, (c) $\mathrm{S}_{13} \mathrm{I}_{7}$, (d) $\mathrm{S}_{14} \mathrm{I}_{6}$, (e) $\mathrm{S}_{15} \mathrm{I}_{5}$, (f) $\mathrm{S}_{16} \mathrm{I}_{4}$, (g) $\mathrm{S}_{17} \mathrm{I}_{3}$, (h) $\mathrm{S}_{18} \mathrm{I}_{2}$ and (i) $\mathrm{S}_{19} \mathrm{I}_{1}$, respectively. The order parameters were calculated using an executable, namely isosueface.exe, that is an implemented tool in DL_MESO software. Yellow and violet beads represent coarse-grained beads of styrene and isoprene, respectively, Table S1: Parameters used in DPD simulation in dimensionless unit, Script S1: Structural knowledge analysis and PCA visualization.

Author Contributions: Conceptualization, methodology, software, validation, N.C. and P.N.; formal analysis, investigation, resources, data curation, writing - original draft preparation, visualization, N.C., M.A. and P.N.; writing-revision and editing, N.C., M.A., K.M., V.S.L., S.J. and P.N.; supervision, project administration, funding acquisition, P.N. All authors have read and agreed to the published version of the manuscript.

Funding: P.N. and V.S.L. acknowledge visiting professor grants from Chiang Mai University (CMU) (Grant Number R000026614). N.C. gratefully thanks the Science Achievement Scholarship of Thailand (SAST) for partial financial support.

Institutional Review Board Statement: Not applicable.

Informed Consent Statement: Not applicable.

Data Availability Statement: Not applicable.

Acknowledgments: The authors would like to acknowledge the computer resources from the Computational Simulation and Modeling Laboratory (CSML) and Quantum-Atom Optics Laboratory (QAO) at CMU. N.C. acknowledges the Graduate School, CMU.

Conflicts of Interest: The authors declare no conflict of interest.

\section{References}

1. Schacher, F.H.; Rupar, P.A.; Manners, I. Functional block copolymers: Nanostructured materials with emerging applications. Angew. Chem. Int. Ed. 2012, 51, 7898-7921. [CrossRef] [PubMed]

2. Orilall, M.C.; Wiesner, U. Block copolymer based composition and morphology control in nanostructured hybrid materials for energy conversion and storage: Solar cells, batteries, and fuel cells. Chem. Soc. Rev. 2011, 40, 520-535. [CrossRef]

3. Khandpur, A.K.; Foerster, S.; Bates, F.S.; Hamley, I.W.; Ryan, A.J.; Bras, W.; Almdal, K.; Mortensen, K. Polyisoprene-polystyrene diblock copolymer phase diagram near the order-disorder transition. Macromolecules 1995, 28, 8796-8806. [CrossRef]

4. Förster, S.; Khandpur, A.K.; Zhao, J.; Bates, F.S.; Hamley, I.W.; Ryan, A.J.; Bras, W. Complex phase behavior of polyisoprenepolystyrene diblock copolymers near the order-tisorder transition. Macromolecules 1994, 27, 6922-6935. [CrossRef]

5. Chiangraeng, N.; Keyen, U.; Yoshida, N.; Nimmanpipug, P. Temperature-responsive morphology formation of PS-b-PI copolymer: A dissipative particle dynamics simulation study. Soft Matter 2021, 17, 6248-6258. [CrossRef]

6. Chiangraeng, N.; Lee, V.S.; Nimmanpipug, P. Coarse-grained modelling and temperature effect on the morphology of PS- $b$-PI copolymer. Polymers 2019, 11, 1008. [CrossRef] [PubMed] 
7. Weiyu, C.; Tashiro, K.; Hanesaka, M.; Takeda, S.; Masunaga, H.; Sasaki, S.; Takata, M. First detection of lamella-gyroid-cylinder phase transition of neat polyethylene-poly(ethylene oxide) diblock copolymers on the basis of synchrotron WAXD/SAXS and infrared/Raman spectral measurements. J. Phys. Conf. Ser. 2009, 184. [CrossRef]

8. Jain, S.; Dyrdahl, M.H.E.; Gong, X.; Scriven, L.E.; Bates, F.S. Lyotropic Phase Behavior of Poly(ethylene oxide)-Poly(butadiene) Diblock Copolymers: Evolution of the Random Network Morphology. Macromolecules 2008, 41, 3305-3316. [CrossRef]

9. Weiyu, C.; Tashiro, K.; Hanesaka, M.; Takeda, S.; Masunaga, H.; Sasaki, S.; Takata, M. Relationship between morphological change and crystalline phase transitions of polyethylene-poly(ethylene oxide) diblock copolymers, revealed by the temperaturedependent synchrotron WAXD/SAXS and infrared/raman spectral measurements. J. Phys. Chem. B 2009, 113, $2338-2346$. [CrossRef]

10. Cochran, E.W.; Garcia-Cervera, C.J.; Fredrickson, G.H. Stability of the gyroid phase in diblock copolymers at strong segregation. Macromolecules 2006, 39, 2449-2451. [CrossRef]

11. Fraaije, J.G.E.M.; van Vlimmeren, B.A.C.; Maurits, N.M.; Postma, M.; Evers, O.A.; Hoffmann, C.; Altevogt, P.; Goldbeck-Wood, G. The dynamic mean-field density functional method and its application to the mesoscopic dynamics of quenched block copolymer melts. J. Chem. Phys. 1997, 106, 4260-4269. [CrossRef]

12. Fraaije, J.G.E.M. Dynamic density functional theory for microphase separation kinetics of block copolymer melts. J. Chem. Phys. 1993, 99, 9202-9212. [CrossRef]

13. Chiangraeng, N.; Lee, V.S.; Nimmanpipug, P. Phase separation dynamics and morphologies prediction of PEO- $b$-PMMA copolymer by atomistic and mesoscopic simulations. J. Met. Mater. Miner. 2018, 28, 18-26. [CrossRef]

14. Seaton, M.A.; Anderson, R.L.; Metz, S.; Smith, W. DL_MESO: Highly scalable mesoscale simulations. Mol. Simul. 2013, 39, 796-821. [CrossRef]

15. Prinsen, P.; Warren, P.B.; Michels, M.A.J. Mesoscale Simulations of Surfactant Dissolution and Mesophase Formation. Phys. Rev. Lett. 2002, 89, 148302. [CrossRef] [PubMed]

16. Warren, P.B.; Prinsen, P.; Michels, M.A.J. The physics of surfactant dissolution. Philos. Trans. R. Soc. Lond. Ser. A 2003, 361, 665-676. [CrossRef]

17. Jolliffe, I.T. Principal Component Analysis, 2nd ed.; Springer: New York, NY, USA, 2002; ISBN 0-387-95442-2.

18. Abdi, H.; Williams, L.J. Principal component analysis. Wiley Interdiscip. Rev. Comput. Stat. 2010, 2, 433-459. [CrossRef]

19. Kar, R.K.; Bhunia, A. Will it be beneficial to simulate the antifreeze proteins at ice freezing condition or at lower temperature? J. Phys. Chem. B 2015, 119, 11485-11495. [CrossRef]

20. Fernandez, M.; Trefiak, N.R.; Woo, T.K. Atomic property weighted radial distribution functions descriptors of metal-organic frameworks for the prediction of gas uptake capacity. J. Phys. Chem. C 2013, 117, 14095-14105. [CrossRef]

21. Humphrey, W.; Dalke, A.; Schulten, K. VMD: Visual molecular dynamics. J. Mol. Graph. 1996, 14, 33-38. [CrossRef]

22. Ayachit, U. The ParaView Guide: A Parallel Visualization Application; Kitware Inc.: New York, NY, USA, 2015; ISBN 978-1930934306.

23. R Core Team. A Language and Environment for Statistical Computing; R Foundation for Statistical Computing: Vienna, Austria, 2021; Available online: http:/ / www.r-project.org/ (accessed on 13 June 2021).

24. Kassambara, A.; Mundt, F. Factoextra: Extract and Visualize the Results of Multivariate Data Analyses. R Package Version 1.4.1717. 2021. Available online: https:/ / cran.r-project.org/package=factoextra (accessed on 15 June 2021).

25. R Package "Corrplot": Visualization of a Correlation Matrix. (Version 0.89). Available online: https://github.com/taiyun/corrplot (accessed on 15 June 2021).

26. Li, X.; Guo, J.; Liu, Y.; Liang, H. Microphase separation of diblock copolymer poly(styrene-b-isoprene): A dissipative particle dynamics simulation study. J. Chem. Phys. 2009, 130, 074908. [CrossRef] [PubMed]

27. Hajduk, D.A.; Harper, P.E.; Gruner, S.M.; Honeker, C.C.; Kim, G.; Thomas, E.L.; Fetters, L.J. The gyroid: A new equilibrium morphology in weakly segregated diblock copolymers. Macromolecules 1994, 27, 4063-4075. [CrossRef]

28. Lodge, T.P.; Pudil, B.; Hanley, K.J. The full phase behavior for block copolymers in solvents of varying selectivity. Macromolecules 2002, 35, 4707-4717. [CrossRef] 